Aus der Chirurgischen Universitätsklinik in Rostock. (Direktor: Geh.-Rat Müller.)

\title{
Ueber die Störung der Salzsäuresekretion des Magens bei Erkrankung der Gallenblase.
}

\author{
Von Dr. M. Behm, Assistenzarzt der Klinik.
}

Nach den zuerst von Holl $\mathrm{H}_{\mathrm{w}} \mathrm{g}$ und Rost angestellten Untersuchungen bestelit bei Cholezystitis und Cholelithiasis außerordentlich häufig eine verminderte sekretorische Funktion des Magens. Den gleichen Befund stellten d:...elben Autoren bei der Nachuntersuchung cholezystektomierter Patienten fest. Während neuerdings $\mathrm{Lamér}$ is und Rohde bei der Untersuchung von Gallenblasenkranken vor und nach der Cholezystektomie ebenfalls häufig eine An- oder Hypazidität des Mageninhaltes fanden, werden diese Resultate von dem Dänen Mogens Fenger, welcher die Magensäurewerte vor und nach der Cholezystektomie untersuchte, nicht bestätigt. Auch $\mathrm{Kehr}$, Grube and Graff hatten bei Cliolelithiasis, besonders bei Choledochusverschlluß, häufig Hyperazidität gefundell. Diese einander widersprechenden Befunde ließen es angezeigt erscheinen, gelegentlich der Nachuntersuchung von Patienten, welche in Laufe der letzten Jahre in der hiesigen Klinik wegen Gallenblasenerkrankungen operiert wurden, die Aufmerksamkeit besonders auf die sekretorischel1 Verhältnisse des Magens zu lenken und auf etwaige mit diesen in Zusammenhang stehende Beschwerden zu achten.

Wir schlossen Patienten, die über 50 Jahre alt waren, von vornherein von den Untersuchungen aus, da nach Ueberschreiteri dieser Altersgrenze ill 30-40\% der Fälle hypazide Werte des Magens gefundell werden, ohne diß subjektive Beschwerden oder objektive Krankheitssymptonı seitens des Magens bestelıen (S e idelin, Li ef schüt z). Wir stellten zunächst die Mageninhaltsuntersuchungen nach Probefrühstück von 49 Patienten, welche vor der Operation vorgenommell wurden, zusammen. Dabei ist besonders zu betonen, daB diese Untersuchungen fast ausnahmslos aus der Vorkriegszeit stammen. Es kommt somit die Frage der durch, die Kriegsernährung zum Teil verursachten Kriegshypazidität für diese nicht in Betracht. Es fanden sich

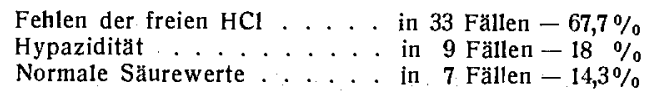

In keinem Fall bestand Hyperazidität. Bei 23 von diesen vor der Operation untersuchten Fällen ergab der autoptische Befund der

1) Kraus-Brugsch, Hb., Lieferung $48-53,97-98,104-109 .-{ }^{2}$ ) Die „Nutzdos isa in $10 \mathrm{~cm}$ Tiefe beträgt unter diesen Bedingungen bei Fn-Filterung ca $28 \%$, bei AlFilterung etwa $19 \%$ der Oberflächen davon. Allerdings können wir diese Messungen nur an Fürstenauschen Wasserphantom mit dem Intensimeter ausführen. Die HED etreichen wir unter $\mathrm{Fn}$ in ca 50 , unter $\mathrm{Al}$ in etwa 25 Minuten. - ${ }^{3}$ ) In v. Domarus, Klin. Hämatol., Thieme 1919. 
Operation einen völligen Verschluß des Ductus cysticus odcr eille hochgradige Schrumpfung der Gallenblase und somit einen totalen Funktionsausfall der Gallenblase. Es bestand bei diesen

$$
\begin{aligned}
& \text { Fehlen der freien } \mathrm{HCl} \ldots \ldots \text { in } 18 \text { Fällen }-78,3 \% \\
& \text { Hypazidität . . . . . . in } 5 \text { Fällen }-21,7 \%
\end{aligned}
$$

in keinem Fall normale oder hyperazide Werte.

$\mathrm{Nach}$ der Operation kamen 22 Patienten zur Nachuntersuchung: bei welchen vor mindestens zwei Jahren die Cholezystektomie, zum Teil mit gleichzeitiger Choledochotomie und Hepatikusdrainage, ausgeführt war. Die Untersuchung des Mageninhalts nach Probefrühstück ergab bei diesen Patienten

Fehlen der freien HCI . . . . in 12 Fällen $-54,5 \%$
Hypazidität . . . . . . . . in 8 Fällen $-36,3 \%$
Normale Säurewerte . . . . in 2 Fällen $-9,2 \%$

Hyperazidität in keinem Fall.

Diese Zahlen zeigen, daß bei Gallenblasenerkrankungen und nach Exstirpation der Gallenblase die freie Salzsäure im Magensaft sehr oft fehlt und fast immer Hypazidität besteht. Unsere Resultate deckeıl sich im wesentlichen mit den zuerst von $\mathrm{Hohlweg}$ angcstelltei Untersuchungen. Es besteht nun die Frage, inwieweit die Untersuchn1119 der Magensekretion Wert hat für die Diagnostik der Gallenblasenerkrankungen. Besonders bei der chronischen Cholezystitis bzw. Cholelithiasis ist der Wert der Mageninhaltsuntersuchung unverkenmbar in der Sicherung der Differentialdiagnose gegenüber dem juxtapylorischen Ulcus ventriculi und dem Ulcus duodeni. Bei beiden letzteren $\mathrm{Er}$ krankungen findet sich doch in der überwiegenden Mehrzahl der Fälle Hyperchlorhydrie des Magens. So spricht bei der häufig nicht eindeutigen Ânamnese und dem klinisch geringen Befunde be der chronischen Verlaufsform der Erkrankung der Gallenblase wie des dem Pylorus benachbarten Ulkus An- bzw. Hypazidität entschieden eher für Cholezystitis, Hyperazidität für Ulkus. Der diagnostische Wert der Mageninhaltsuntersuchung besteht um so mehr, als bei den chronischen Fällen beider Erkrankungen auch die Röntgendurchleuchtung häufig kein eindeutiges Resultat ergibt. Besonders bei der Diagnose des Ulcus duodeni läßt der Röntgenschirm oft genug im Stich. Anderseits bestehen bei Gallenblasenerkrankungen, wie Rohd e berichtet und wie auch wir häufig zu beobachten Gelegenheit habeı, oft röntgenologische Veränderungen des Magens in Gestalt von Verziehungen des Pylorus und Verzerrungen und Trichterbildungen im Bereich del oberen Abschnitte des Duodenums, welche durch Ad häsionen und pericholezystitische Prozesse verursacht sind.

Zur Frage des Einflusses des lkterus auf die sckiretorische Funktion des Magens ist Folgendes zu bemerken: Siminsky fand bei Ikterus verschiedener Herkunft fast stets Hyperchlorhydrie des Magcns. Wir konnten das nicht bestätigen. Unter den oben erwähnten 49 vor der Operation untersuchten Fällen fand sich $8 \mathrm{mal}$ lkterus infolge von Konkrementen in den Gallenwegen. Es bestand 5mal Anazidität, 3mal Hypazidität des Mageninhaltes.

Bei dem Ergebnis der vor der Operation untersuchten Fatienten verdient noch folgende Tatsache besondere Beachtung. Die Prozentzahl des Fehlens der freien Salzsäure ist bei derjenigen Reihe von Patienten am höchsten $(78,3 \%)$, bei welchen der autoptische Befund der Operation einen Verschluß des Ductus cysticus oder eine hochgradige Schrumpfung der Gallenblase ergab und so einen totalen Funktionsausfall derselben erwies. Das entspricht den Befunden von Hohlweg und Rohde, welche ebenfalls bei völligem Funktionsausfall der Gallenblase am häufiusten ( $\mathrm{Hohlw}$ eg in $84 \%$, Rohde in $83,35 \%$ der untersuchtell Fälle) Fehlen der freien Salzsäure beobachtet hatten.

Diese Herabsetzung der Salzsäuresekretion bleibt nun bei weitaus der Mehrzahl der cholezystektomierten Patienten dauend bestehen. Man muß demnach Wechselwirkungen zwischen den Sekretionsverhältnissen des Magens und der veränderten Art der Gallensekretion annehmen, wie sie sich nach Ausschaltung der Gallenblase vollzieht. Hier kommen mechanische, chemische und reflektorische Ursachen in Frage. Rost hat durch experimentelle Untersuchungen an Hunden, denen er die Gallenblase exstirpiert und eine Duodenalfistel angelegt hatte, gefunden, daß bei einem Teil der Tiere die dünne Lebergalle dauernd abtropfte, während bei den übrigen sich eine relative Kontinenz der Papille wieder einstellte und die Galle periodisch auf den Reiz der eingeführten Nahrung entleert wurde. Trotz dieser Wiederanpassung an physiologische Verhältnisse wiesen diese Hunde ebenso ein Salzsäuredefizit des Magcns auf wie die Hunde mit dem kontinuierlichen Gallenabfluß. Es müssen also außer letzterem, welcher durch die dauernde Berührung der Duodenalschleimhaut mit der alkalischeń Galle reflektorisch die Magensekretion hemmt, noch andere, bisher unbekannte Momente in Betrach gezogen werden, welche die Hypofunktion des Magenchemismus bewirken. Vielleicht spielt dabei die Stanung der Galle, weIche in den Gallenwegen nach Entfernung der bis dahin als Druckregulator wirkenden Gallenblase eintritt, eine Rolle

Diese Erwägungen über die funktionelle Bedeutung der Gallenblase haben die Frage nach der Wahl der Operationsmethode nie ganz verstunmen lassen. Wenn auch die Cholezystektomie heute als die Operation der Wahl gilt, so sind doch einige Autoren, so Capelle und neuerdings Rovsing und Borchgrevink --- letztere gerade auf Grund der experimentellen Untersuchungen von Rost -.. soweit es möglich ist, für konservative Operationsmethoden eingetreten.
Unter diesem Gesichtspunkt erhob sich nun die Frage, ob sich ach einer die Gallenblase erhaltenden Operation deren Funktion wieder einstellt und $o b$, wenn dies del Fall ist, der Magenchemismus wieder normal wird. Oder mit anderen Worten: kann daraus, daß ein mit einer konservativen Operationsmethode behandelter Patient einen normalen Magenchemismus wieder erlangt, geschlossen werden, daß auch die Gallenblase ihre Funktion wieder übernommen hat? All der hiesigen Klinik ist seit dem Jahre 1913 die Cholezystektomie die Operation der Wahl geworden, während bis zu diesem Zeitpunkt die Cholezystostomie in den Fällen vorgezogen wurde, in denen die Entzündung der Giallenblase nicht zu weit vorgeschritten war und in denen sich die Konkremente auch aus deı tiefereı Gallenwegen mit möglichster Sicherheit entferıen ließen. An diesen cholezystostomierten Patienten stellten wir ebenfalls Nachuntersuchungen an. Die Zahl derselben wurde durch Unauffindbarkeit mancher vor 10 bis 15 Jahren operierter Patienten und durch deıl Umstand begrenzt, daß mancher von ihnen inzwischen das 50. Lebensjahr erreicht hatte. Aus dem oben näher ausyeführten Grunde der Altershypazidität schlossen wir auch diese Patienten von der Untersuchung aus. Immerhin stellten sich 19 Patienten zur Nach. untersuchung ein. Bei diesen fanden sich

Fehlen der freien $\mathrm{HCl} \ldots \ldots$. . . in 9 Fällen $-47,3 \%$
Hypazidität . . . . . . . in 8 Fällen $-42,1 \%$
Normale Säurewerte . . . . in 2 Fällen $-10,6 \%$

Dicsc Zahlen entsprechen durchaus unseren Nachuntersuchungen nach Cholezystektomie. Hier wie dort in der überwiegenden Mehrzahl der Fälle eine Hypofunktion des Magenchemismus. Es ist daher die Schlußfolgerung nicht von der Hand zu weisen, daß auch nach einer die Gallenblase erhaltenden Operation die Funktion derselben sich meist nicht wieder herstellt. Das stimmt durchaus mi den Befunden überein, welche bei 8 Zweitoperationen - Cholezystektomic - - welche im Laufe der letzten 12 Jahre wegen Wiederauftreten von Beschwerden nach Cholezystostomie in der hiesigen Klinik vorgenommen werden mußten, erhoben wurden. In allen diesen Fällen fand sich die Gallenblase durch noch vorhandene Konkreınente, durch Füllung mit einem serösen oder hämorrhagischen Exsudat oder durch ausgedehnte pericholezystische Verwachsungen so hochgradig verändert, daß sie ihrer physiologischell Funktion sicher nicht mehr nachkommen konnte.

Subjektive Beschwerden, welche mit der heraboesetzten Saizsäureabscheidung des Magens in Zusammenhang gebracht werden konnten, bestanden nur bei wenigen der nachuntersuchten Patienten, und zwar sowohl bei den mit konservativer wie bei den mit radikaler Operationsmethode Behandelten. Diese Beschwerden, welche sich im wesentlichen als Druckgefühl in der Magengegend nach dem Essen äußerten, dürften zum Teil auch als Verwachsungsbeschwerden zu deuten seisı.

Zusammenfassung. 1. Bei Gallenblasenerkrankungeı besteht meistens eine Störung der Salzsäuresekretion des Magens. Diese Tatsache hat, besonders bei chronischen Fällen, differentialdiagnostische Bedeutung.

2. Nach Cholezystektomie und ebenso nach Cholezystostomie bleibt die verminderte Salzsäureabscheidung des Magens meist be stehen. Die Gallenblase erlangt also ihre physiologische Funktion auch nach einer konservativen Operation meist nicht wieder.

3. Diese Sekretionsstörung des Magens verursacht keine wesentlichen subjektiven Beschwerden. 